\title{
Lower pole of patella fractures treated with non-absorbable trans-osseous sutures: an effective surgical method to avoid implant related complications
}

\author{
Aniruddha Mondal' ${ }^{1}$, Ayon Das ${ }^{2 *}$
}

${ }^{1}$ Department of Orthopaedics, NRS Medical College and Hospital, Kolkata, West Bengal, India
${ }^{2}$ Department of Orthopaedics, IPGMER and SSKM Hospital, Kolkata, West Bengal, India

Received: 05 March 2021

Revised: 25 March 2021

Accepted: 26 March 2021

\section{*Correspondence:}

Dr. Ayon Das,

E-mail: dr.ayondas@gmail.com

Copyright: () the author(s), publisher and licensee Medip Academy. This is an open-access article distributed under the terms of the Creative Commons Attribution Non-Commercial License, which permits unrestricted non-commercial use, distribution, and reproduction in any medium, provided the original work is properly cited.

\begin{abstract}
Background: The treatment option for inferior pole of patella fracture is still being debated. Although tension-band wiring is the most widely used technique to treat patellar fractures, metal implant-related complications such as implant failure, palpable hardware are very common and additional procedures are often necessary to treat the complications. The aim of the study was to evaluate the functional results in patients with inferior pole of patella fracture treated by trans-osseous non-absorbable suture fixation.

Methods: Patients who underwent trans-osseous suture fixation by no. 5 Ethibond for post-traumatic distal pole patella fracture were included in the study. This was a prospective study conducted at a tertiary care Government Hospital in Kolkata, between December 2018 to May 2020. All patients were followed up at an interval of 2 weeks, 6 weeks, 12 weeks, 6 months and 9 months. Bostman score was used to assess the functional outcomes of the patients.

Results: Outcome at final follow-up was assessed with Bostman scoring system. In a total of 11 patients, 8 (72.8\%) patients showed excellent and $3(27.2 \%)$ patients had good results during the final follow-up. None of the patients demonstrated unsatisfactory result. At the end of 9 months, the average Bostman score for 11 patients was 27.9.

Conclusions: The trans-osseous suturing with non-absorbable sutures is a safe and effective fixation technique for the treatment of distal pole patella fractures. It allows for rapid recovery with minimal implant-related complications. It also reduces the re-surgery rates significantly.
\end{abstract}

Keywords: Inferior pole, Patella fracture, Trans-osseous suturing, Suture repair, Non-metallic fixation

\section{INTRODUCTION}

The patella forms an important constituent of the extensor mechanism of knee by increasing its lever arm. ${ }^{1}$ It is the largest sesamoid bone and its fracture accounts for approximately $1 \%$ of all fractures in adults. ${ }^{2,3}$

Patella inferior pole fractures are extraarticular and constitute $9-22 \%$ of all patellar fractures, which are often associated with complete disruption of the extensor mechanism. ${ }^{4,5}$ Lower pole fractures of patella are commonly avulsion injury due to unexpected sudden flexion of knee joint against a violent contraction of the quadriceps muscle.

Fracture of the distal pole of patella usually are less than $1.5 \mathrm{~cm}$ in vertical height, extra-articularly located and most of the time comminuted. Because of this, treating this type of fracture poses a special problem. ${ }^{6,7}$

There are various previously defined surgical fixation options for patella inferior pole fractures: anterior tension band wiring, combining screw fixation with tension band, patellotibial cerclage, fixed angle plate application, 
separate vertical wiring, basket plate, patellar shapememory fixator applications and excision of the lower pole of patella..$^{8-11}$

Treatment goal of any modality should be restoring extensor mechanism and early mobilization. Furthermore, articular congruity is essential to reduce the increased risk of posttraumatic osteoarthritis as a result of the highcontact forces in the patellofemoral joint. ${ }^{12}$ Now a days, various methods have been advocated for the management these fractures ranging from excision of lower pole with patellar tendon repair to various forms of internal fixation.

Internal fixation of the distal part, if possible, or excision of the small bone fragments with repair of the patellar tendon by trans-osseous pull-out sutures, in the extremely comminuted cases, should be performed. However, in the latter, non-absorbable synthetic sutures and partial patellectomy necessitate immobilization of the knee, which causes weakness in the quadriceps muscle. Therefore, fixation of the distal patellar fractures has gained popularity over partial patellectomy.

Even though substantially good clinical results have been reported, all techniques using metallic wires can result in symptomatic hardware, which represents the most frequent complication, with reported rates varying from 0 to $60 \% .{ }^{13,14}$ In fact, implants can irritate overlying soft tissue and cause pain, requiring a second surgery to remove the implant. In addition, a high incidence of infection has been reported. ${ }^{15}$ For this reason, some authors have advocated the use of nonabsorbable sutures, such as braided polyester, to fix the fracture when using various techniques. ${ }^{16}$

Several advantages of using a suture over a wire have been shown in the literature, such as lower rate of revision surgery and higher patient tolerance of surgical material that does not irritate soft tissues as much as wire. Another advantage is represented by easier handling of suture materials for the surgeon, which could determine shorter operating and tourniquet time. ${ }^{17,18}$

The objective of this study was to represent our experience comprising cases of distal pole of patella fracture which were treated by trans-osseous suture fixation using nonabsorbable sutures to see technique achieves better knee function without facing the implant related complications.

\section{METHODS}

This study was performed in accordance with the ethical standards of the institutional review board. 11 patients with post traumatic lower pole of patella fracture who were treated by trans-osseous non absorbable suture fixation in the Department of Orthopaedics, NRS Medical College and Hospital, Kolkata from December 2018 to May 2020 and fulfilling the inclusion criteria were considered in this study.

\section{Inclusion criteria}

Inclusion criteria included patients above 18 years of age who are skeletally mature, sustained a post-traumatic lower pole of patella fracture, closed injury and fresh fracture ( $<1$ week old).

\section{Exclusion criteria}

Exclusion criteria included open wound, any other bone fracture of ipsilateral lower limb, active infection, previous ipsilateral knee surgery and patients with polytrauma or head injuries that definitely influence rehabilitation.

\section{Operative procedure}

The patients were subjected to a thorough history, clinical examination and pre-operative routine laboratory investigations, which was supplemented by radiographs in antero-posterior and lateral view of the knee joint.

All the patients were operated under spinal anaesthesia. Patients were positioned supine on a radiolucent operating table and pneumatic tourniquet was used.

A standard midline longitudinal skin incision from superior pole of patella to tibial tuberosity was given. After rising flaps fracture ends, torn retinaculum and patellar tendon were exposed. Saline irrigation was done to clear hematoma and clots. Denuded free bony fragments were removed but most of the bone stock was kept intact. Two vertical drill holes were made using cannulated drill bit through the articular margin of proximal fragment and directed to the superior border of patella keeping the knee in flexion. Patellar tendon was sutured by no. 5 Ethibond via Krackow technique weaving through medial and lateral half of patellar tendon, four strands of sutures was passed transosseously with beath pin (two on either side), next sutures held on the superior pole of patella and knee flexion checked up to $90^{\circ}$ before tying a knot. With the knee held in extension, sutures were secured onto the superior pole patella. The patellar tendon was slightly everted and made to lie against the raw fractured surface of the patellar remnants near articular surface. The torn retinaculum was repaired with absorbable sutures. On table knee flexion was checked and to see if any gaping of the repair. Surgical wound was closed in layers. Sterile dressings were applied to the surgical incisions and a long knee brace was applied.

Post-operatively, the patient was encouraged to do isometric quadriceps exercises and ankle pumps from day 1. No knee motion was allowed for the first 4 weeks. Patients were allowed weight bearing as tolerated with the help of bilateral axillary crutches on knee immobilizer. Range of motion and quadriceps and hamstring strengthening exercises were started at 4 weeks following surgery and crutches were gradually weaned off. The immobilizer was discontinued after 6 weeks. All the rehabilitation was done under the direct supervision of a physiotherapist. 
The patients were regularly followed up for 9 months (2 weeks, 6 weeks, 12 weeks, 6 months and 9 months) for clinical as well as radiological evaluation.

\section{Statistical analysis}

The data was collected in Microsoft excel (Windows 10; version 2016) and statistical software SPSS version 20 was used for analysis. Procedure of the data analysis was transcription, preliminary data inspection, content analysis and interpretation. The categorical variables like age, sex, side, mode of injury was expressed as number of patients and to differentiate using the mean scores a non-parametric Wilcoxon matched paired test was used at 0.05 level of significance.

\section{RESULTS}

\section{Age distribution}

The mean age in this study was 29.8 years. The youngest patient was 20 years old and the eldest patient was 44 years old. Overall, $81.8 \%$ patients were between the age group of 20-40 years. It might be because younger peoples are more active and involved in outdoor and sports activities which makes them more prone to injuries.

Table 1: Age distribution.

\begin{tabular}{|llll|}
$\begin{array}{l}\text { Age } \\
\text { (years) }\end{array}$ & $\begin{array}{l}\text { No. of } \\
\text { patients }\end{array}$ & $\begin{array}{l}\text { Percentage } \\
(\%)\end{array}$ & P value \\
\hline $\mathbf{1 8 - 3 0}$ & 5 & 45.4 & \\
\hline $\mathbf{3 1 - 4 0}$ & 4 & 36.4 & 0.001 \\
\hline $\mathbf{> 4 0}$ & 2 & 18.2 & \\
\hline Total & 11 & 100.0 & \\
\hline
\end{tabular}

\section{Sex distribution}

In study, 9 patients $(81.8 \%)$ were male and 2 patients were female $(18.2 \%)$. Majority of patients were male which may be because of more outdoor and sports related activities makes them more vulnerable to accidents and trauma.

\section{Side of injury distribution}

In this study, number of right knee involvement was 7 $(63.6 \%)$ which was higher in comparison with left knee involvement which was $4(36.4 \%)$.

\section{Mode of injury distribution}

In this study, $6(54.5 \%)$ patients suffered a fall, $3(27.3 \%)$ were involved in road traffic accident and 2 (18.2\%) sustained trauma during sports activities.

\section{Post-operative final range of motion distribution}

Among 11 patients, $9(81.8 \%)$ patients had $0-130^{\circ} \mathrm{ROM}$, $1(9.1 \%)$ patient had $0-120^{\circ} \mathrm{ROM}$ and $1(9.1 \%)$ patient 0 $110^{\circ}$ ROM by the end of 9 months.
Table 2: Post-operative final range of motion distribution.

\begin{tabular}{|c|c|c|c|}
\hline $\begin{array}{l}\text { Final ROM } \\
\text { (in degree) }\end{array}$ & $\begin{array}{l}\text { No. of } \\
\text { patients }\end{array}$ & $\begin{array}{l}\text { Percentage } \\
(\%)\end{array}$ & P value \\
\hline $0-130$ & 9 & 81.8 & \multirow{4}{*}{0.001} \\
\hline 0-120 & 1 & 9.1 & \\
\hline $0-110$ & 1 & 9.1 & \\
\hline Total & 11 & 100.0 & \\
\hline
\end{tabular}

\section{Bostman score}

At the end of 9 months, the average Bostman score for 11 patients was 27.9.

\section{Bostman score outcome}

In a total of 11 patients, $8(72.8 \%)$ patients showed excellent and $3(27.2 \%)$ patients had good results at the end of 9 months follow-up. None of the patients demonstrated unsatisfactory result.

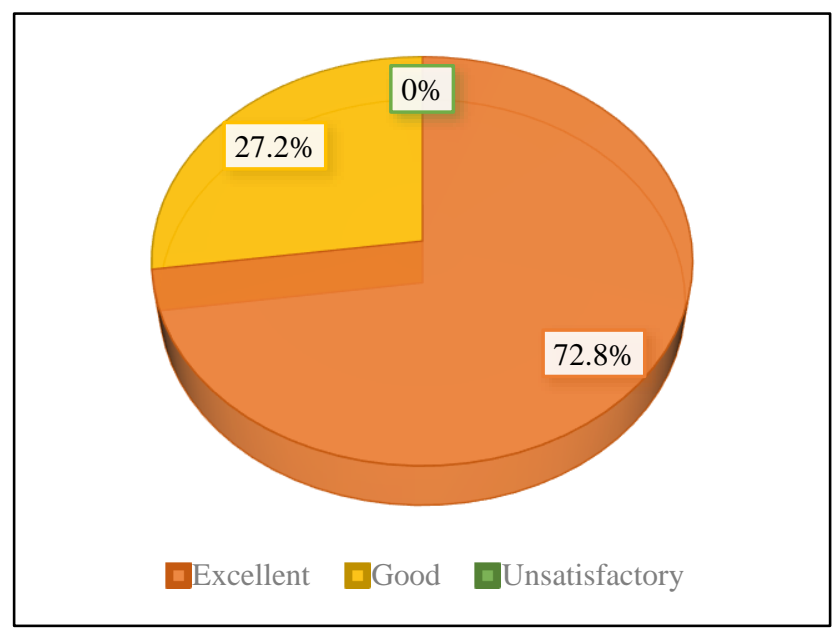

Figure 1: Bostman score outcome.

\section{Complications}

Out of 11 patients, $10(90.9 \%)$ patients did not have any complications. Only $1(9.1 \%)$ patient developed a superficial wound infection which subsided with a course of intravenous antibiotic for 10 days. Minor complications such as pain, knee stiffness was observed initially during the course of the study but all of them finally settled by the end of 9 months follow up. There were no reports of rerupture or suture pull out or Patella baja during the entire study period. None of the patients had flexion deformity or extensor lag at final follow up. There were no hardware related complications encountered in the course of the study. None of the patients presented with symptoms of discomfort secondary to implant that required re-surgery.

Majority of patients, 7 (63.6\%), presented within 1 week of injury. All patients had a positive extensor lag before surgery. The average time to radiological union was observed to be almost 3.5 months. 


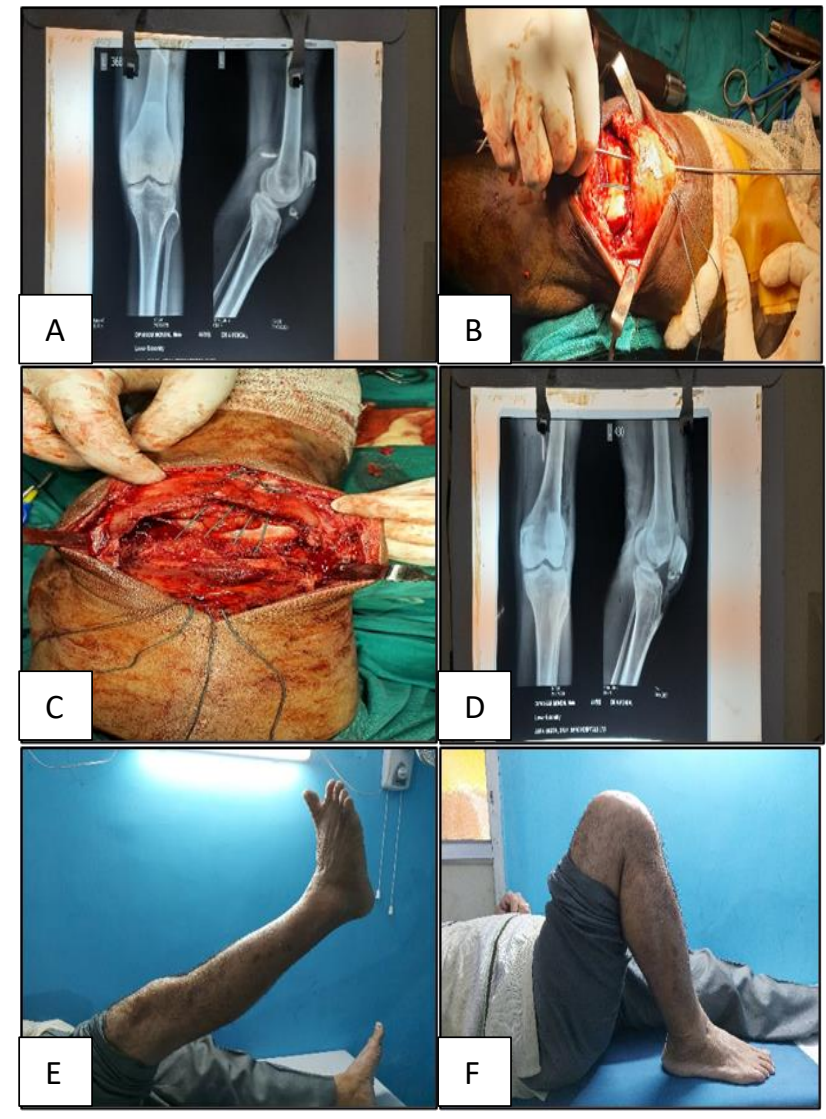

Figure 2: (A) Pre-op X-ray of lower pole of patella fracture; (B) passing of beath pin through proximal fragment; (C) passage of trans-osseous sutures; (D) immediate post-op X-ray of reduced and apposed fracture fragments; (E) knee extension at the final follow up; (F) knee flexion at the final follow up.

\section{DISCUSSION}

The inferior pole patella is an anterior cortical extension and is devoid of articular cartilage. Many studies about resection of distal pole have proved clinically and biochemically that there is shortening of lever arm of extension mechanism. ${ }^{19}$

With various methods described for treating such fractures none has yet been advocated as ideal method. There is a re-operation rate between $20 \%$ and $50 \%$ when inferior pole fractures are fixed with K-wires. ${ }^{7}$ In many of these reoperated knees, restriction of knee joint range of motion developed. The number of implants related metallic complications were very high in these patients as there was a high incidence of K-wire migration and the stainlesssteel wire knots that were not buried properly produced skin irritation, ulcers and infections. These complications ranged from $0-60 \% .^{20}$ All these factors played an important role in influencing the removal of the implant.

There is no consensus regarding the ideal treatment of patella inferior pole fractures. Currently, new surgical approaches for the optimal management are still under development. The aim of such surgical treatment is to achieve stable fixation with restoration of the patellar tendon length, which permits early postoperative motion. ${ }^{21,22}$

In our study we used no. 5 Ethibond suture material which is non-absorbable, has high molecular weight, is a long chain linear polyester and features unique braid configuration. No. 5 Ethibond suture material also offers high knot breaking strength, and superior strength.

Gosal et al performed a study comparing two groups for patella fracture fixation, one metallic group and the other a non-metallic group and concluded that the metallic group had 6 times higher infection, re-surgery and morbidity rates when compared to the non-absorbable polyester group and hence the study supported the use of nonmetallic implants for patella fractures. ${ }^{23}$

According to case report by Huang et al, fixation of inferior pole with $\mathrm{K}$-wire leads to re-operation rates between $20 \%$ to $50 \%$ and further restriction of knee joint range of motion develops. The inherent weakness of the bone and the size of fragments prevent rigid fixation by ordinary wiring or screws. ${ }^{24}$

Egol et al conducted a retrospective cohort study in 49 patients with patella fracture and concluded that patients who sustain inferior pole patella fractures have limited options for fracture fixation. Suture repair is clinically acceptable, yielding similar results to patella fractures repaired with metal implants. Importantly, patients undergoing suture repair appear to have fewer hardware related postoperative complications than those receiving wire fixation for mid pole fractures. ${ }^{25}$

Buezo et al described a new surgical procedure among 8 patients consisting of open reduction followed by internal fixation by performing 3 longitudinal tunnels crossing double high-resistance sutures within these tunnels and suturing among them and the result being safe and with similar functional outcomes when compared with other techniques. Furthermore, no second surgeries for implant removal were required during their study. ${ }^{26}$

Srikant et al conducted a prospective study on 12 patients and found that partial patellectomy and patellar tendon repair using trans-osseus sutures is very effective method in the management of comminuted extraarticular inferior pole patella fractures. ${ }^{27}$ At the end of 6 months, all patients had successful outcomes and returned to their pre-injury activity level. There was no case that required reoperation.

Limitations of our study include single institution bias, small group of patients, short follow-up period and a lack of control group. Additional prospective and biomechanics studies should be conducted to confirm these outcomes in the future. A multicentre study with more patients is essential to substantiate benefits of this treatment method. 


\section{CONCLUSION}

The trans-osseous suturing with non-absorbable suture is a safe, cost effective and effective fixation technique in achieving union in distal pole of patella fractures. This technique can provide strong and stable fixation, enable patients to perform an early functional exercise and has a good clinical effect with excellent functional outcome and low rate of complications. It allows for rapid recovery with minimal implant-related complications such as implant failure and palpable hardware. Removal of implant is not necessary as it does not cause symptomatic implant complications resulting in decreased re-surgery rates.

A larger well-designed study is required to be conducted across the country to corroborate the findings of our study.

Funding: No funding sources

Conflict of interest: None declared

Ethical approval: The study was approved by the institutional ethics committee

\section{REFERENCES}

1. Sutton FS Jr, Thompson CH, Lipke J, Kettelkamp DB. The effect of patellectomy on knee function. J Bone Joint Surg Am. 1976;58:537-40.

2. Kaufer H. Mechanical function of the patella. J Bone Joint Surg Am. 1971;53(8):1551-60.

3. Melvin JS, Mehta S. Patellar fractures in adults. J Am Acad Orthop Surg. 2011;19(4):198-207.

4. Neumann HS, Winckler S, Strobel M. Long-term results of surgical management of patellar fractures. Unfallchirurg. 1993;96:305-10.

5. Chang SM, Ji XL. Open reduction and internal fixation of displaced patella inferior pole fractures with anterior tension band wiring through cannulated screws. J Orthop Trauma. 2011;25:366-70.

6. Fletcher C. Comminuted fractures of the lower pole of the patella-to fix or resect? A case report and review of the literature. EC Orthop. 2015;2.1:54-9.

7. Joshi RR, Dwivedi R, Byanjanakar S, Shrestha R. Outcome of inferior patella pole avulsion fractures: a comparative study. J Lumbini Med College. 2016;4(2):84-9.

8. Kim YM, Yang JY, Kim KC, Kang C. Separate Vertical Wirings for the Extra-articular Fractures of the Distal Pole of the Patella. Knee Surg Relat Res. 2011;23:220-26.

9. Liu X-W, Shang H-J, Xu S-G, Wang Z-W. Patellar shape-memory fixator for the treatment of comminuted fractures of the inferior pole of the patella. $\mathrm{J}$ materials engineering performance. 2011;20:623-8.

10. Thelen S, Betsch M, Schneppendahl J, Grassmann J. Fixation of multi-fragmentary patella fractures using a bilateral fixed-angle plate. Orthopedics. 2013;36:e1437-43.
11. Yang KH, Byun YS. Separate vertical wiring for the fixation of comminuted fractures of the inferior pole of the patella. J Bone Joint Surg Br. 2003;85:1155-60.

12. Carpenter JE, Kasman RA, Patel N, Lee ML, Goldstein SA. Biomechanical evaluation of current patella fracture fixation techniques. J Orthop Trauma. 1997;11:351-6.

13. Appel MH, Seigel H. Treatment of transverse fractures of the patella by arthroscopic percutaneous pinning. Arthroscopy. 1993;9:119-21.

14. Smith ST, Cramer KE, Karges DE, Watson JT, Moed BR. Early complications in the operative treatment of patella fractures. J Orthop Trauma. 1997;11:183-7.

15. Gardner MJ, Griffith MH, Lawrence BD, Lorich DG. Complete exposure of the articular surface for fixation of patellar fractures. J Orthop Trauma. 2005;19:118-23.

16. Chiang CC, Huang CK, Chen WM, Lin CF, Tzeng YH, Liu CL. Arthroscopically assisted percutaneous osteosynthesis of displaced transverse patellar fractures with figure-eight wiring through paired cannulated screws. Arch Orthop Trauma Surg. 2011;131:949-54.

17. Wild M, Thelen S, Jungbluth P. Fixed-angle plates in patella fractures-a pilot cadaver study. Eur J Med Res. 2011;16:41-6.

18. Reider B, Marshall J, Koslin B. The anterior aspect of the knee joint. J Bone Joint Surg Am. 1981;63(A):351-6.

19. Johnson EE. Fractures of the patella. In: Rockwood C, Green D, Bucholz RW, Heckman JD. (eds). Rockwood and Green's fractures in adults ( $4^{\text {th }}$ ed.). Philadelphia: Lippincott-Raven. 1996;1956-72.

20. Carpenter JE, Kasman R, Matthews LS. Fractures of the patella. Instr Course Lect. 1994;43:97-108.

21. Hunt DM, Somashekar N. A review of sleeve fractures of the patella in children. Knee. 2005;12:3-7.

22. Thelen S, Betsch M, Schneppendahl J, Grassmann J. Fixation of multi fragmentary patella fractures using a bilateral fixed-angle plate. Orthopedics. 2013;36:e1437-43.

23. Gosal HS, Singh P, Field RE. Clinical experience of patellar fracture fixation using metal wire or non-absorbable polyester-A study of 37 cases. Injury. 2001;32:129-35.

24. Huang HC, Su JY, Cheng YM. Modified basket plate for inferior patellar pole avulsion fracture - a report of three cases. Kaoshing J Med Sci. 2012;28(11):619-23.

25. Kenneth Egol MD, Daniel Howard BS, Roy Davidovitch BS. Patella Fracture Fixation with Suture and Wire: you reap what you Sew Iowa Orthop J. 2014;34:63-7.

26. Buezo O, Cuscó X, Seijas R, Sallent A, Ares O, Álvarez-Díaz P et al. Patellar fractures: an innovative surgical technique with transosseous suture to avoid implant removal. Surgical innovation. $2015 ; 22(5): 474-8$. 
27. Srikant K, Mishra D, Sinha VK, Pradhan S. Management of comminuted extraarticular inferior pole patella fractures with partial patellectomy and patellar tendon repair by transosseous sutures. Int $\mathbf{J}$ Med Sci Public Health. 2017;6(11):1578-84.
Cite this article as: Mondal A, Das A. Lower pole of patella fractures treated with non-absorbable trans-osseous sutures: an effective surgical method to avoid implant related complications. Int J Res Orthop 2021;7:481-6. 